Muschalla, B. (2016). Different work capacity impairments in patients with different work-anxieties. International Archives of Occupational and Environmental Health, 89, 609-619.

Different work capacity impairments in patients with different work-anxieties

Original article for publication in International Archives of Occupational and Environmental Health

Running head: work capacity impairment in work-anxieties

Author: Dr. phil. Beate Muschalla

Affiliation: Department of Work and Organizational Psychology, University of Potsdam, Germany

Word count: 3908 words (abstract, manuscript text), 3 tables

Funding: This research has been financially supported by the German Pension Fund. 8011 - 106 - 31/31.107.

Conflicts of interest: The author declares that there are no conflicts of interest.

Correspondence address: Beate Muschalla, University of Potsdam, Department of Psychology, Work and Organizational Psychology, Karl-Liebknecht-Straße 24-25, 14476 Potsdam, Germany. Email: beate.muschalla@gmx.de 


\title{
Different work capacity impairments in patients with different work-anxieties
}

\author{
Abstract \\ Purpose \\ Persons with work-anxieties are especially endangered for work-impairment and \\ sick-leave. Work-impairment is not directly due to symptoms but due to illness- \\ related capacity impairments. Work capacity impairments can be described on \\ different dimensions (e.g. social interaction, decision making and judgment, \\ endurance, mobility). Understanding the type of work capacity impairment is \\ important for reintegration interventions This is the first study to investigate \\ work capacity impairment in risk-patients with different work-anxieties.
}

\section{Methods}

Two hundred forty four patients in inpatient rehabilitation suffering from workanxieties were investigated concerning degree of work capacity impairment. Capacity impairment was described on 13 capacity dimensions according to the internationally evaluated observer-rating Mini-ICF-APP (impairment grades 0-4, grade 2 and higher indicating clinically relevant observable impairment). A physician's rating on global work ability prognosis was obtained, and sick-leave duration during six months after assessment. Patients with different workanxieties were compared concerning capacity impairments. 


\section{Results}

Patients with different work-anxieties were impaired in different capacity dimensions: work-related social anxiety went along with clinically relevant impairment in capacity of assertiveness $(M=2.40)$, anxiety of insufficiency went along with impaired capacity of endurance $(M=2.20)$, work-related generalized worrying was accompanied by impairment in the capacity for decision making $(M=1.82)$. Specific capacity impairment dimensions were related with sick-leave duration, while a global work ability prognosis was not.

\section{Conclusions}

The capacity approach is useful to describe work-impairment more precisely and beyond symptoms. On this basis reintegration-focusing interventions such as capacity training (e.g. social interaction training) or work adjustment (e.g. reducing exposure with interactional work tasks) can be initiated.

\section{Key words}

Work ability, work-anxiety, sick leave, impairment, mental disorders, ICF 


\section{Introduction}

Mental disorders impact work ability and are often associated with sick leave (Ekberg et al. 2015; Hensing et al. 2013; Lidwall 2015) or presenteeism (Esposito et al. 2007). Work-anxieties have been found to be especially risky for impaired work ability and sick leave (Muschalla and Linden, 2009). Work ability impairment has often been described in a global way and due to symptoms (e.g. Tuomi et al. 1998). However, healing the symptoms and the mental illness itself is often not a primary aim. Instead, improvement of the capacity status of a person may be possible (Baron and Linden 2009), as well as workplace adjustment referring to the capacity impairments. When describing work capacity, it must be noticed that this comprises different capacities of a person (Baron and Linden 2009), which may be impaired to different degrees. It is until now unknown if, and if so, which work capacities of patients with different workanxieties are impaired.

Therefore, the first aim of the current study is to investigate in which wise patients with different work-anxieties are impaired in different dimensions of work capacity. Work capacity impairment thereby means to which degree a person is impaired in performing circumscribed patterns of activities at work. A second, rather explorative question of research is whether and in which wise work capacity impairments are correlated with later sick leave duration. Results will give hints towards which capacity dimensions may be of special importance when restoring work ability.

Towards a concept of work capacity impairment

Work ability impairment has often been described as resulting from symptoms 
(Dell-Kuster et al. 2014; Huijs et al. 2012; Stansfeld et al. 1995; Szubert and Sobala 2007). However, it has been found that the symptom itself does not predict work ability (Gatchel et al. 1994). The socio-medical work ability decisions (i.e. the medical decision whether the person can go to work or not) cannot be made based on an illness symptom or diagnosis only. They must be based on a description what kind of required activities the person cannot fulfill due to his/her illness (Baron and Linden 2009; Linden et al. 2010; WahlinNorgren et al. 2011). Work ability has often been described globally, in the sense that a person has "problems of keeping up his work performance" to a certain degree (Hannula et al. 2006), or with a distinction into "physical" and "mental" work ability (Work Ability Index WAI, Tuomi et al. 1998). However, work ability is not a homogenous construct: Different jobs require different psychological capacities to a different degree (e.g. Boschman et al. 2014). Furthermore, different capacities of a person may be impaired to different degrees. Therefore different work-relevant psychological capacities should be distinguished in work ability description. In work and organizational research, there are workplace analysis approaches which focus on describing work demands in terms of capacity and activities (e.g. O*Net, Peterson et al. 1999; Fleishman and Reilley 1992). This applies to the approach of person-role-fit (French 1973). Similar to this approach, the bio-psycho-social health model of the International Classification of Functioning Disability and Health ICF (WHO 2001) offers a level of more concretely describing work ability in terms of activities and capacities. When dealing with mental disorders, it must be taken into consideration that mental disorders are usually chronic by their nature and cause problems in work over the life span (Stansfeld et al. 2008). Since symptoms 
mostly do not disappear enduringly, lots of psychotherapeutic interventions are capacity trainings by their nature (Muschalla 2014), e.g. social competency trainings, problem solving, activity planning, and stress management interventions. In socio-medical practice dealing with patients with work-anxieties and mental disorders, an approach for describing work capacity impairment on thirteen dimensions has been developed and until now widely evaluated (MiniICF-APP, Baron and Linden, 2009; Linden et al. 2009).

Work-anxieties and work capacity impairment dimensions One mental health problem which is in a specific risky way related with work ability impairment and sick leave are work-anxieties. About $5 \%$ in mentally healthy working employees (Muschalla et al. 2013) and 30-60\% of patients in rehabilitation clinics suffer from work-anxieties (Linden and Muschalla 2007; Muschalla and Linden 2009, 2013). These patients are in a different way impaired in work ability and especially workplace phobics are prone for long-term sick leave (Muschalla and Linden 2009). Different types of work-anxiety can be distinguished empirically according to the differential diagnostic of anxiety psychopathology (Linden and Muschalla 2007; Muschalla and Linden 2013). As anxiety is partly a stimulus-bound phenomenon, different work-anxieties can be expected to be associated with different work capacity impairments: Social anxieties towards specific colleagues or supervisors may come along with impairment in social activities at work like participation in a team meeting (teamwork capacity), contacts with clients (capacity for contacts with thirds), or expressing voice (assertiveness) towards a colleague or supervisor, or handling aggressive thirds. Work-related hypochondriac anxiety presents in exaggerated 
self-observation and protective behavior and which may become visible in a dysfunctional (exaggerated) self-care, eventually accompanied by impairment in flexibility and in endurance (due to the protective behavior and avoiding working fast and hard). Patients with work-related anxiety of insufficiency may have difficulties in applying their expertise, or coping with the amount of work. This may become visible in impairment in endurance. Patients with workplace phobia are expected to show impairment in mobility, i.e. the capacity to come to the workplace. This is because workplace phobic persons get physiological arousal symptoms when approaching their workplace (Haines et al. 2002) and thus they aim to avoid the workplace. They are also prone to have longer sick leave durations than patients with other work-anxieties or general mental disorders (Muschalla and Linden 2009). Patients with work-related generalized worrying (Shearer and Gordon 2006) may have special problems in the cognitive capacity of decision making and judgment, as their thoughts are focused on potentially upcoming negative events.

Until now it is unclear in which wise different work-anxieties are accompanied by different work capacity impairments. To clear these empirical relations, patients with work-anxieties have been investigated concerning their work capacity impairments. Next, the relationship between capacity impairment and sick leave duration has been explored. 


\section{Method}

Study sample

1610 consecutively admitted patients (aged 18-65) were approached in the beginning of their inpatient rehabilitation treatment in a screening interview on work-anxieties (Muschalla et al. 2014). 828 reported an elevated score of workanxiety in the screening and 429 agreed to participate in a more detailed diagnostic interview. From those, 393 patients fulfilled the criteria of at least one work-anxiety according to the Work-Anxiety-Interview (Linden and Muschalla 2007; Muschalla and Linden 2009). About half (49.8 \%) of them had one single work-anxiety diagnosis, $43.6 \%$ had two or three and $6.6 \%$ had four up to six work-anxiety diagnosis. These comorbidities are normally occurring when using structured diagnostic interviews. Two hundred forty four patients could be included in the analysis with complete diagnostic data: They were diagnosed with DSM-based structured diagnostic interviews on mental disorders (Sheehan et al. 1994) and work-anxieties (Linden and Muschalla 2007), and physicians' sociomedical work ability prognosis was available, as well as a follow-up information on the patients sick leave duration six months after rehabilitation. Patients included in the analysis were on average $50.23(S D=27.9)$ years of age. $53.3 \%$ were women. $73.6 \%$ had a completed apprenticeship, $20.7 \%$ a university diploma, $2.6 \%$ had a master craftman's certificate, $2.2 \%$ had no completed professional education and $0.9 \%$ were still in education. The great majority $(84.4$ \%) presently obtained a workplace. Concerning their present or last professional status, $24.7 \%$ were employed blue-collar workers, $4 \%$ unskilled workers, $45.8 \%$ white-collar employees, $18.5 \%$ employees with leading position, $6.2 \%$ were 
self-employed. Concerning medical impairments, $27.8 \%$ had a medically certified chronic disability, $4.4 \%$ had applied for disability pension, The average sick leave duration in the past twelve months was $M=8.64(S D=14.4)$ weeks; $60.8 \%$ of the participants had been on sick leave in the past 12 months. Beside the work-anxiety diagnosis, $72.2 \%$ of this sample had a general mental disorder according to DSM (33) in their medical history, mostly affective disorders (e.g. depressive episodes in $20.7 \%, 4.8 \%$ reported lifetime attempt in their history), anxiety disorders (6.2\% panic, $13.2 \%$ agoraphobia, $26 \%$ generalized anxiety disorder) ${ }^{1}$.

This analysis is part of a larger research project including a capacityoriented group intervention for helping persons with work-anxieties return to work (Muschalla et al. 2014). The study was approved by the ethics committee of the University of Potsdam. The study was done between May 2012 and July 2014.

Instruments

Diagnosis of mental disorders for the purpose of population description were assessed with the DSM-based internationally evaluated and established Mini International Neuropsychiatric Interview MINI (Sheehan et al. 1994) covering the full range of the common mental disorders.

The Work-Anxiety-Interview (Linden and Muschalla 2007; Muschalla and Linden

\footnotetext{
${ }^{1}$ Diagnosis of general mental disorders were equally distributed in the patients with different work-anxieties. Only in eight out of 126 comparisons some diagnoses were overrepresented: 13 patients with workplace-phobia had a depressive episode, 11 had an agoraphobia, and 2 an addiction problem. From patients with work-related anxiety of insufficiency, 27 had a depressive episode, and 12 a social phobia. From patients with work-related social phobia, 10 had a personality disorder. From patients with work-related situational anxiety, 12 had a
} 
2009) was done for differential diagnostic of work-anxieties. It covers the different psychopathological categories of work-anxieties, i.e. work-related posttraumatic stress disorder, situational anxiety, anxiety of insufficiency, social anxiety, hypochondriac anxiety, adjustment disorders with anxiety, generalized worrying, and workplace phobia. The interview had been validated with different anxiety-self-rating questionnaires (Linden and Muschalla 2007; Muschalla and Linden 2013) and has good inter-rater reliabilities of kappa $=0.97$ (two independent raters). All diagnoses are coded with "yes" (diagnosis stated) or "no" (no diagnosis).

The differentiated description of work ability impairment in terms of capacities was operationalized with the Mini-ICF-APP. The Mini-ICF-APP capacity rating is a short observer rater instrument for the description of (work) capacity impairment. It is internationally validated (Balestrieri et al. 2013; Baron and Linden 2009; Linden et al. 2015; Molodynski et al. 2013) and has become a recommended instrument in sociomedical work ability description (DGPM 2012; DRV 2012; Schneider et al. 2012; SGVP 2012). The Mini-ICF-APP covers thirteen dimensions of psychological capacities which are often impaired due to mental disorders: Adherence to regulations, planning and structuring of tasks, flexibility, applying expertise, capacity to judge and decide, endurance, assertiveness, contacts with others, teamwork capacity, self-care, mobility, and the non-work domains of non-work recreational activities, familiar and intimate relationships. For quantifying capacity impairment, a concrete reference context is needed. In this present study capacity impairment is rated in respect to the reference context "work", i.e. the present workplace or the professional field in which the person (if presently unemployed) could find an occupation according to his/her qualification level. The impairment rating was done after collecting information on the work context and 
respective work demands, as well as illness-related impairments concerning these work demands in a structured interview (Linden et al. 2015). The interview can be done in about 45 minutes. For each capacity dimension, the impairment degree is rated $0=$ no impairment, $1=$ mild impairment, i.e. there are some difficulties for the person to fulfill the demands but there are no negative consequences, 2 = relevant impairment, i.e. there are visible problems in fulfilling the demands, $3=$ severe impairment, i.e. help from others is needed regularly in order to fulfill the demands and activities, $4=$ full impairment, i.e. no respective activity is possible, complete dispensation is necessary. The rating is a clinical expert rating, i.e. the interviewer has to rate the degree of impairments on the basis of his observation and the information on capacity impairment explored from the patient. Therefore the interview questions must be posed precisely behavior-oriented in order to get the relevant information on work activity and capacity impairment (instead of wellbeing impairment) from the patient. The rater in this study is a state licensed psychotherapist with ten years of expertise in psychosomatic rehabilitation diagnostic and socio-medical work ability description. A trained psychological co-rater assisted in a part of the interviews $(n=46)$ for proving inter-rater-reliability. The inter-rater reliability of the capacity impairment ratings from the interviewer and the co-rater in this study was between $r=.706$ (endurance) and $r=.940$ (mobility). This speaks for adequate process objectivity (doing the interview and rating according to the manual), and therefore rater bias can be expected to be limited.

Additionally to the thorough capacity assessment, the treating physicians gave their judgment on the temporal work ability concerning the present or past workplace and for general work ability, i.e. work ability for the general labor market. They had to judge how many hours a day the patient is able to work: under 3 hours (0), 3 up to six hours (3) or 
more than six hours (6).

Finally, the patients were asked for the cumulative duration of sick leave in a follow-up six months after inpatient rehabilitation. Patients reported their six leave time in weeks.

Statistical analysis

Data analysis were performed using the IBM SPSS statistics program version 22. Separate multivariate analysis (MANCOVA) were calculated for comparison of capacity impairments in patients with a respective specific work-anxiety and patients with other work-anxieties. For each separate calculation it was controlled for influences of the respective other work-anxieties. Means and standard deviations of the capacity impairments are reported, and levels of effects sizes (partial eta-squared) and significant differences between the groups. Correlations between capacity impairment and work-anxieties, and correlations between capacity impairment and later sick leave duration were calculated for explorative purpose.

\section{Results}

The correlations between the capacity impairment dimensions ranged between $r=$ .119 (intimate relationships and endurance) and $r=.707$ (teamwork capacity and contacts). 68 out of 78 correlations were below $r=.50$. There was no high correlation (above $r=.80$ ) between any of the capacity dimensions. Thus the (unavoidable) phenomenon of multicollinearity should not be a serious problem in these data. The capacity dimensions can be seen as rather distinct dimensions. 
Table 1 shows the results from the multivariate comparisons of the capacity impairments in the different work-anxieties. Overall, highest capacity impairments at a clinically relevant level (a score of "2" indicating relevant impairment, i.e. there are observable problems in fulfilling the work demands) were found in endurance $(M=1.80,60 \%$ have observable problems $)$ and flexibility $(M=1.70,59 \%$ have observable problems). Lowest impairment was found in self-care. This rank order of capacity impairment was similar to findings from comparable other study samples (Balestrieri et al. 2013; Baron and Linden 2009). 
Table 1 MANCOVA Multivariate analysis of variance with covariates. Patients with specific work-anxiety diagnosis are compared to patients with other work-anxiety-diagnosis (WA) concerning their degree of capacity impairments $(N=222)$. Analyses for each work-anxiety have been calculated controlling for the respective six "other" work-anxieties. Pilai's trace overall multivariate statistics are reported in the first lines. Means (standard deviations), and significance level $p$ and effect size [partial eta-squared] of comparison are reported for each capacity dimension and the socio-medical data physicians prognosis and sick leave duration. Percentages of patients with capacity impairment of " 2 " or higher (rating 0-4).

\begin{tabular}{|c|c|c|c|c|c|c|c|c|c|c|c|c|c|c|c|}
\hline \multirow[t]{2}{*}{$\begin{array}{l}\text { Degree of } \\
\text { capacity } \\
\text { impairment }\end{array}$} & \multicolumn{2}{|c|}{$\begin{array}{l}\text { Adjustment } \\
\text { Disorder with } \\
\text { Anxiety }\end{array}$} & \multicolumn{2}{|c|}{$\begin{array}{c}\text { Specific Social } \\
\text { Anxieties }\end{array}$} & \multicolumn{2}{|c|}{$\begin{array}{l}\text { Situational } \\
\text { Anxiety }\end{array}$} & \multicolumn{2}{|c|}{$\begin{array}{l}\text { Hypochondriac } \\
\text { Anxiety }\end{array}$} & \multicolumn{2}{|c|}{$\begin{array}{l}\text { Generalized } \\
\text { Worrying }\end{array}$} & \multicolumn{2}{|c|}{$\begin{array}{c}\text { Anxiety of } \\
\text { Insufficiency }\end{array}$} & \multicolumn{2}{|c|}{$\begin{array}{l}\text { Workplace } \\
\text { Phobia }\end{array}$} & \multirow[t]{2}{*}{$\begin{array}{c}\text { Any } \\
\text { Work- } \\
\text { Anxiety }\end{array}$} \\
\hline & With & $\begin{array}{c}\text { With } \\
\text { other } \\
\text { WA } \\
n=194\end{array}$ & With & $\begin{array}{c}\text { With } \\
\text { other } \\
\text { WA } \\
n=162\end{array}$ & With & $\begin{array}{c}\text { With } \\
\text { other } \\
\text { WA } \\
n=139\end{array}$ & With & $\begin{array}{c}\text { With } \\
\text { other } \\
\text { WA } \\
n=205\end{array}$ & With & $\begin{array}{c}\text { With } \\
\text { other } \\
\text { WA } \\
n=138\end{array}$ & With & $\begin{array}{c}\text { With } \\
\text { other } \\
\text { WA } \\
n=161\end{array}$ & With & $\begin{array}{c}\text { With } \\
\text { other } \\
\text { WA } \\
n=196\end{array}$ & \\
\hline $\begin{array}{l}\text { Pilai's trace } \\
\mathrm{F}\left(\mathrm{df}_{\text {hyp }}, \mathrm{df} \text { err }\right) \\
\text { Significance } p \\
\text { [Partial eta- }\end{array}$ & \multicolumn{2}{|c|}{$\begin{array}{c}1.16(16,199) \\
.304 \\
{[.085]}\end{array}$} & \multicolumn{2}{|c|}{$\begin{array}{c}4.37(16,199) \\
.000 \\
{[.260]}\end{array}$} & \multicolumn{2}{|c|}{$\begin{array}{c}2.39(16,199) \\
.003 \\
{[.161]}\end{array}$} & \multicolumn{2}{|c|}{$\begin{array}{c}1.18(16,199) \\
.290 \\
{[.086]}\end{array}$} & \multicolumn{2}{|c|}{$\begin{array}{c}2.71(16,199) \\
.000 \\
{[.262]}\end{array}$} & \multicolumn{2}{|c|}{$\begin{array}{c}4.42(16,199) \\
.001 \\
{[.179]}\end{array}$} & \multicolumn{2}{|c|}{$\begin{array}{c}2.29(16,19 \\
.004 \\
{[.156]}\end{array}$} & \\
\hline $\begin{array}{l}\text { Adherence to } \\
\text { regulations }\end{array}$ & $\begin{array}{l}0.86 \\
(0.97) \\
{[.000]} \\
26.7 \%\end{array}$ & $\begin{array}{c}0.81 \\
(1.18)\end{array}$ & $\begin{array}{l}1.02 \\
(1.08) \\
{[.005]} \\
31.7 \%\end{array}$ & $\begin{array}{c}0.74 \\
(1.18)\end{array}$ & $\begin{array}{c}0.67 \\
(0.89) \\
{[.002]} \\
17.7 \%\end{array}$ & $\begin{array}{c}0.89 \\
(1.29)\end{array}$ & $\begin{array}{l}1.29 \\
(1.16) \\
{[.008]} \\
58.9 \%\end{array}$ & $\begin{array}{c}0.78 \\
(1.15)\end{array}$ & $\begin{array}{c}0.64 \\
(0.86) \\
{[.014]} \\
18.6 \%\end{array}$ & $\begin{array}{c}0.92 \\
(1.30)\end{array}$ & $\begin{array}{l}1.10 * \\
(1.58) \\
{[.022]} \\
25.1 \%\end{array}$ & $\begin{array}{c}0.71 \\
(0.93)\end{array}$ & $\begin{array}{l}1.38 * \\
(1.09) \\
{[.019]} \\
46.5 \%\end{array}$ & $\begin{array}{c}0.74 \\
(1.14)\end{array}$ & $\begin{array}{c}0.76 \\
(0.93)\end{array}$ \\
\hline $\begin{array}{l}\text { Planning and } \\
\text { structuring } \\
\text { tasks }\end{array}$ & $\begin{array}{l}1.00 \\
(0.90) \\
{[.000]} \\
26.7 \%\end{array}$ & $\begin{array}{c}0.94 \\
(0.91)\end{array}$ & $\begin{array}{l}1.00 \\
(0.96) \\
{[.000]} \\
23.8 \%\end{array}$ & $\begin{array}{c}0.93 \\
(0.89)\end{array}$ & $\begin{array}{l}0.86 \\
(0.91) \\
{[.000]} \\
212 \%\end{array}$ & $\begin{array}{l}1.00 \\
(0.90)\end{array}$ & $\begin{array}{c}1.35 \\
(1.11) \\
{[.016]} \\
47 \%\end{array}$ & $\begin{array}{c}0.91 \\
(0.88)\end{array}$ & $\begin{array}{l}1.12 * \\
(0.81) \\
{[.028]} \\
32.6 \%\end{array}$ & $\begin{array}{c}0.84 \\
(0.95)\end{array}$ & $\begin{array}{l}1.23 * \\
(0.88) \\
{[.041]} \\
391 \%\end{array}$ & $\begin{array}{c}0.84 \\
(0.89)\end{array}$ & $\begin{array}{l}1.35^{*} \\
(0.85) \\
{[.021]} \\
357 \%\end{array}$ & $\begin{array}{c}0.89 \\
(0.90)\end{array}$ & $\begin{array}{c}0.95 \\
(0.91)\end{array}$ \\
\hline Flexibility & $\begin{array}{l}2.00 \\
(0.90) \\
{[.009]} \\
73.3 \%\end{array}$ & $\begin{array}{l}1.65 \\
(0.93)\end{array}$ & $\begin{array}{l}1.78 \\
(0.90) \\
{[.000]} \\
60.3 \%\end{array}$ & $\begin{array}{l}1.67 \\
(0.94)\end{array}$ & $\begin{array}{l}1.59 \\
(0.96) \\
{[.001]} \\
54.3 \%\end{array}$ & $\begin{array}{c}1.77 \\
(1.91)\end{array}$ & $\begin{array}{l}2.18 \\
(1.04) \\
{[.017]} \\
70.5 \%\end{array}$ & $\begin{array}{l}1.66 \\
(0.92)\end{array}$ & $\begin{array}{l}1.82 \\
(0.85) \\
{[.013]} \\
65.2 \%\end{array}$ & $\begin{array}{l}1.63 \\
(0.97)\end{array}$ & $\begin{array}{l}1.85 \\
(1.89) \\
{[.013]} \\
65.7 \%\end{array}$ & $\begin{array}{l}1.65 \\
(1.94)\end{array}$ & $\begin{array}{l}2.31 \% \\
(0.79) \\
{[.039]} \\
85.8 \%\end{array}$ & $\begin{array}{c}1.62 \\
(0.92)\end{array}$ & $\begin{array}{l}1.70 \\
(0.94)\end{array}$ \\
\hline $\begin{array}{l}\text { Applying } \\
\text { expertise }\end{array}$ & $\begin{array}{l}1.50 \\
(1.04)\end{array}$ & $\begin{array}{c}1.13 \\
(1.16)\end{array}$ & $\begin{array}{l}1.27 \\
(1.13)\end{array}$ & $\begin{array}{l}1.15 \\
(1.15)\end{array}$ & $\begin{array}{c}1.01 \\
(1.18)\end{array}$ & $\begin{array}{l}1.28 \\
(1.12)\end{array}$ & $\begin{array}{l}1.47 \\
(1.33)\end{array}$ & $\begin{array}{l}1.16 \\
(1.13)\end{array}$ & $\begin{array}{l}1.12 \\
(0.99)\end{array}$ & $\begin{array}{c}1.22 \\
(1.23)\end{array}$ & $\begin{array}{l}1.46^{*} \\
(1.13)\end{array}$ & $\begin{array}{c}1.07 \\
(1.14)\end{array}$ & $\begin{array}{l}2.00^{*} \\
(0.98)\end{array}$ & $\begin{array}{c}1.07 \\
(1.13)\end{array}$ & $\begin{array}{c}1.17 \\
(1.14)\end{array}$ \\
\hline
\end{tabular}




\begin{tabular}{|c|c|c|c|c|c|c|c|c|c|c|c|c|c|c|c|}
\hline & {$[.006]$} & & {$[.000]$} & & {$[.003]$} & & {$[.001]$} & & {$[.002]$} & & {$[.023]$} & & {$[.053]$} & & \\
\hline & $43.3 \%$ & & $28.1 \%$ & & $22.5 \%$ & & $47.1 \%$ & & $28 \%$ & & $39.1 \%$ & & $67.9 \%$ & & $31 \%$ \\
\hline $\begin{array}{l}\text { Capacity to } \\
\text { judge and }\end{array}$ & $\begin{array}{c}1.64 \\
(0.91)\end{array}$ & $\begin{array}{c}1.50 \\
(0.98)\end{array}$ & $\begin{array}{c}1.53 \\
(0.95)\end{array}$ & $\begin{array}{c}1.51 \\
(0.98)\end{array}$ & $\begin{array}{c}1.34 \\
(0.99)\end{array}$ & $\begin{array}{c}1.63 \\
(0.94)\end{array}$ & $\begin{array}{c}1.94 \\
(0.97)\end{array}$ & $\begin{array}{c}1.81 \\
(1.03)\end{array}$ & $\begin{array}{l}1.82 * * \\
(0.75)\end{array}$ & $\begin{array}{c}1.33 \\
(1.04)\end{array}$ & $\begin{array}{l}1.74^{*} \\
(0.89)\end{array}$ & $\begin{array}{c}1.43 \\
(0.98)\end{array}$ & $\begin{array}{l}2.19 * * \\
(0.75)\end{array}$ & $\begin{array}{c}1.43 \\
(0.96)\end{array}$ & $\begin{array}{c}1.51 \\
(0.97)\end{array}$ \\
\hline decide & {$[.000]$} & & {$[.002]$} & & {$[.001]$} & & {$[.010]$} & & {$[.068]$} & & {$[.020]$} & & {$[.065]$} & & \\
\hline & $63.3 \%$ & & $57.2 \%$ & & $45.9 \%$ & & $64.7 \%$ & & $70.6 \%$ & & $61 \%$ & & $82.8 \%$ & & $53.9 \%$ \\
\hline Endurance & 2.04 & 1.79 & 1.82 & 1.83 & $1.99 *$ & 1.73 & 1.94 & 1.48 & 1.71 & 1.89 & $2.20 * *$ & 1.68 & 2.15 & 1.78 & 1.83 \\
\hline & $(0.88)$ & (1.04) & $(1.07)$ & $(1.00)$ & $(0.97)$ & (1.04) & $(0.97)$ & $(0.96)$ & $(1.02)$ & $(1.02)$ & $(0.98)$ & $(1.00)$ & $(0.92)$ & (1.03) & (1.03) \\
\hline & {$[.015]$} & & {$[.000]$} & & {$[.038]$} & & {$[.004]$} & & {$[.002]$} & & {$[.076]$} & & {$[.011]$} & & \\
\hline & $69 \%$ & & $56.6 \%$ & & $65.5 \%$ & & $64.7 \%$ & & $56.5 \%$ & & $74.8 \%$ & & $74 \%$ & & $60.3 \%$ \\
\hline Assertiveness & 1.82 & 1.41 & $2.40 * *$ & 1.11 & 1.17 & 1.63 & 1.41 & 1.46 & $1.29 *$ & 1.57 & $1.72 *$ & 1.36 & $2.31 *$ & 1.35 & 1.46 \\
\hline & $(1.25)$ & $(1.20)$ & $(0.89)$ & (1.13) & (1.09) & $(1.25)$ & $(0.87)$ & $(1.23)$ & $(1.25)$ & $(1.18)$ & (1.17) & $(1.21)$ & $(1.23)$ & $(1.16)$ & $(1.22)$ \\
\hline & {$[.002]$} & & {$[.190]$} & & {$[.011]$} & & {$[.002]$} & & {$[.020]$} & & {$[.021]$} & & {$[.031]$} & & \\
\hline & $60 \%$ & & $85.8 \%$ & & $38.8 \%$ & & $41.2 \%$ & & $44.3 \%$ & & $56.2 \%$ & & $75 \%$ & & $48.4 \%$ \\
\hline Contacts with & 0.78 & 0.67 & $1.03 *$ & 0.56 & $0.39 *$ & 0.86 & 0.76 & 0.68 & $0.45 * *$ & 0.83 & $1.07 *$ & 0.54 & 0.88 & 0.66 & 0.68 \\
\hline others & $(0.96)$ & $(0.97)$ & $(1.00)$ & $(0.96)$ & $(0.83)$ & $(1.01)$ & (1.03) & $(0.97)$ & $(0.79)$ & (1.04) & $(1.11)$ & $(0.87)$ & $(1.07)$ & $(0.96)$ & $(0.96)$ \\
\hline & {$[.001]$} & & {$[.035]$} & & {$[.040]$} & & {$[.000]$} & & {$[.058]$} & & {$[.050]$} & & {$[.000]$} & & \\
\hline & $26.6 \%$ & & $31.7 \%$ & & $11.8 \%$ & & $29.4 \%$ & & $16.3 \%$ & & $39 \%$ & & $28.6 \%$ & & $22.3 \%$ \\
\hline Teamwork & 0.93 & 0.88 & $1.45^{* *}$ & 0.67 & $0.52 *$ & 1.10 & 1.00 & 0.87 & $0.61 * *$ & 1.05 & 1.15 & 0.78 & 1.27 & 0.83 & 0.88 \\
\hline capacity & $(1.02)$ & $(1.15)$ & (1.19) & (1.04) & $(0.82)$ & $(1.24)$ & (1.17) & $(1.13)$ & $(0.85)$ & $(1.25)$ & $(1.21)$ & (1.09) & $(1.22)$ & (1.11) & (1.13) \\
\hline & {$[.001]$} & & {$[.071]$} & & {$[.054]$} & & {$[.000]$} & & {$[.060]$} & & {$[.013]$} & & {$[.003]$} & & \\
\hline & $26.7 \%$ & & $41.2 \%$ & & $16.5 \%$ & & $35.3 \%$ & & $19.7 \%$ & & $36 \%$ & & $39.2 \%$ & & $26.6 \%$ \\
\hline Self-care & 0.32 & 0.39 & 0.37 & 0.40 & $0.17^{*}$ & 0.52 & $0.88^{*}$ & 0.35 & 0.41 & 0.38 & $0.67^{*}$ & 0.23 & $0.77^{*}$ & 0.34 & 0.40 \\
\hline & $(0.61)$ & $(0.74)$ & $(0.76)$ & $(0.71)$ & $(0.46)$ & $(0.81)$ & $(1.05)$ & $(0.67)$ & $(0.71)$ & $(0.73)$ & $(0.85)$ & $(0.63)$ & $(1.03)$ & $(0.66)$ & $(0.76)$ \\
\hline & {$[.003]$} & & {$[.005]$} & & {$[.030]$} & & {$[.022]$} & & {$[.000]$} & & {$[.047]$} & & {$[.032]$} & & \\
\hline & $10 \%$ & & $12.7 \%$ & & $4.7 \%$ & & $35.3 \%$ & & $11.7 \%$ & & $18.8 \%$ & & $28.5 \%$ & & $10 \%$ \\
\hline Mobility & 0.64 & 0.50 & 0.72 & 0.44 & 0.51 & 0.53 & 0.53 & 0.52 & 0.59 & 0.47 & $0.43^{*}$ & 0.75 & $1.15^{*}$ & 0.43 & 0.54 \\
\hline & $(1.03)$ & $(0.95)$ & (1.11) & $(0.89)$ & (0.98) & $(0.95)$ & (1.07) & $(0.95)$ & $(0.79)$ & $(0.95)$ & $(0.88)$ & (1.12) & $(1.26)$ & $(0.88)$ & $(0.98)$ \\
\hline & {$[.000]$} & & {$[.010]$} & & {$[.006]$} & & {$[.000]$} & & {$[.008]$} & & [.029] & & {$[.053]$} & & \\
\hline & $20 \%$ & & $20.2 \%$ & & $16.3 \%$ & & $11.8 \%$ & & $18.6 \%$ & & $18.7 \%$ & & $32.1 \%$ & & $14.8 \%$ \\
\hline Familiar and & 0.78 & 0.62 & 0.65 & 0.64 & 0.51 & 0.72 & 0.88 & 0.62 & 0.79 & 0.55 & 0.70 & 0.61 & 0.85 & 0.61 & 0.65 \\
\hline intimate & $(0.99)$ & $(0.86)$ & $(0.89)$ & $(0.88)$ & $(0.85)$ & $(0.89)$ & $(1.05)$ & $(0.86)$ & $(0.95)$ & $(0.83)$ & $(0.82)$ & $(0.90)$ & $(0.78)$ & $(0.89)$ & $(0.91)$ \\
\hline
\end{tabular}




\begin{tabular}{|c|c|c|c|c|c|c|c|c|c|c|c|c|c|c|c|}
\hline relationships & {$[.002]$} & & {$[.000]$} & & {$[.004]$} & & {$[.004]$} & & {$[.014]$} & & {$[.001]$} & & {$[.004]$} & & 3060 \\
\hline $\begin{array}{l}\text { Non-work } \\
\text { activities }\end{array}$ & $\begin{array}{c}0.96 \\
(1.08) \\
{[.013]} \\
26.6 \%\end{array}$ & $\begin{array}{c}0.66 \\
(0.85)\end{array}$ & $\begin{array}{c}0.80 \\
(0.97) \\
{[.002]} \\
23.8 \%\end{array}$ & $\begin{array}{c}0.67 \\
(0.85)\end{array}$ & $\begin{array}{c}0.61 \\
(0.87) \\
{[.004]} \\
18.8 \%\end{array}$ & $\begin{array}{c}0.76 \\
(0.89)\end{array}$ & $\begin{array}{l}1.12 * \\
(1.11) \\
{[.021]} \\
29.4 \%\end{array}$ & $\begin{array}{c}0.67 \\
(0.86)\end{array}$ & $\begin{array}{l}0.88 * \\
(0.92) \\
{[.034]} \\
23.3 \%\end{array}$ & $\begin{array}{c}0.59 \\
(0.84)\end{array}$ & $\begin{array}{l}1.00 * \\
(0.97) \\
{[.056]} \\
26.6 \%\end{array}$ & $\begin{array}{c}0.59 \\
(0.83)\end{array}$ & $\begin{array}{l}1.19 * \\
(1.02) \\
{[.028]} \\
32.2 \%\end{array}$ & $\begin{array}{c}0.64 \\
(0.85)\end{array}$ & $\begin{array}{c}0.70 \\
(0.88)\end{array}$ \\
\hline $\begin{array}{l}\text { Specific } \\
\text { workplace- } \\
\text { related work } \\
\text { ability } \\
\text { prognosis by } \\
\text { physician }\end{array}$ & $\begin{array}{c}3.96 \\
(2.71) \\
{[.002]}\end{array}$ & $\begin{array}{c}4.45 \\
(2.56)\end{array}$ & $\begin{array}{c}4.00 \\
(2.74) \\
{[.004]}\end{array}$ & $\begin{array}{c}4.54 \\
(2.51)\end{array}$ & $\begin{array}{c}4.41 \\
(2.54) \\
{[.003]}\end{array}$ & $\begin{array}{c}4.38 \\
(2.61)\end{array}$ & $\begin{array}{c}4.76 \\
(2.38) \\
{[.003]}\end{array}$ & $\begin{array}{c}4.36 \\
(2.59)\end{array}$ & $\begin{array}{c}4.68 \\
(2.39) \\
{[.007]}\end{array}$ & $\begin{array}{c}4.22 \\
(2.68)\end{array}$ & $\begin{array}{c}4.03 \\
(2.79) \\
{[.009]}\end{array}$ & $\begin{array}{c}4.53 \\
(2.49)\end{array}$ & $\begin{array}{c}3.35 \\
(2.97) \\
{[.017]}\end{array}$ & $\begin{array}{c}4.53 \\
(2.49)\end{array}$ & $\begin{array}{c}4.40 \\
(2.57)\end{array}$ \\
\hline $\begin{array}{l}\text { General work } \\
\text { ability } \\
\text { prognosis by } \\
\text { physician }\end{array}$ & $\begin{array}{c}5.04 \\
(2.01) \\
{[.002]}\end{array}$ & $\begin{array}{c}5.30 \\
(1.76)\end{array}$ & $\begin{array}{c}5.10 \\
(2.01) \\
{[.002]}\end{array}$ & $\begin{array}{c}5.33 \\
(1.71)\end{array}$ & $\begin{array}{c}5.20 \\
(1.82) \\
{[.000]}\end{array}$ & $\begin{array}{c}5.31 \\
(1.78)\end{array}$ & $\begin{array}{c}5.65 \\
(0.99) \\
{[.004]}\end{array}$ & $\begin{array}{c}5.24 \\
(1.84)\end{array}$ & $\begin{array}{c}5.50 \\
(1.46) \\
{[.008]}\end{array}$ & $\begin{array}{c}5.13 \\
(1.95)\end{array}$ & $\begin{array}{l}4.87 * \\
(2.20) \\
{[.023]}\end{array}$ & $\begin{array}{c}5.42 \\
(1.59)\end{array}$ & $\begin{array}{c}4.73 \\
(2.43) \\
{[.010]}\end{array}$ & $\begin{array}{c}5.34 \\
(1.68)\end{array}$ & $\begin{array}{c}5.30 \\
(1.76)\end{array}$ \\
\hline $\begin{array}{l}\text { Sick leave } \\
\text { duration } \\
\text { within six } \\
\text { months after } \\
\text { rehabilitation }\end{array}$ & $\begin{array}{c}11.46 \\
(10.50) \\
{[.014]}\end{array}$ & $\begin{array}{c}14.89 \\
(12.50)\end{array}$ & $\begin{array}{c}13.65 \\
(13.23) \\
{[.005]}\end{array}$ & $\begin{array}{c}14.76 \\
(11.95)\end{array}$ & $\begin{array}{c}14.20 \\
(12.72) \\
{[.002]}\end{array}$ & $\begin{array}{c}14.61 \\
(12.08)\end{array}$ & $\begin{array}{c}13.47 \\
(11.67) \\
{[.005]}\end{array}$ & $\begin{array}{c}14.57 \\
(12.36)\end{array}$ & $\begin{array}{c}12.61 \\
(10.85) \\
{[.015]}\end{array}$ & $\begin{array}{c}15.59 \\
(13.00)\end{array}$ & $\begin{array}{c}15.10 \\
(10.94) \\
{[.000\}}\end{array}$ & $\begin{array}{c}14.22 \\
(12.79)\end{array}$ & $\begin{array}{c}18.58 * \\
(10.53) \\
{[.023]}\end{array}$ & $\begin{array}{c}13.91 \\
(12.43)\end{array}$ & $\begin{array}{c}14.51 \\
(12.10)\end{array}$ \\
\hline
\end{tabular}

Notes: ${ }^{*} p<.05,{ }^{* *} p<.01$ indicate significant differences between patients with a respective specific work-anxiety (left column) as compared to patients with other work-anxieties (right column). 
Patients with work-related social anxiety had clinically relevant impairments in the interactional capacities, especially in assertiveness $(M=2.40(S D=0.89)$, indicating obviously observable problems). Patients with generalized worrying had a relevant higher impairment in capacity to judge and decide $(M=1.82(S D=$ 0.75)). Impairment in endurance was high in several work-anxieties, but especially patients with anxiety of insufficiency were even more strongly impaired $(M=2.20(S D=0.98))$. Patients with workplace phobia, different from the other work-anxieties, had a relatively higher impairment in mobility, i.e. the capacity to reach the workplace and move around in the working setting.

Workplace phobic patients were the only group who had significantly longer durations of sick leave in the aftermaths $(M=18.58$ weeks $(S D=10.53))$ than patients with any other work-anxieties but no workplace phobia.

Concerning the two overall socio-medical work ability ratings given by the treating physicians (workplace-related work ability and general work ability), there were no differences between any specific work-anxiety in comparison to the respective other work-anxieties.

Table 2 shows the inter-correlations between capacity impairments and work-anxieties. Social anxieties were significantly related with higher impairment in interactional capacities. Generalized worrying went along with stronger problems in decision making and judgment. Workplace phobia was systematically related with different capacity impairment dimensions, but especially with mobility. Patients with situational anxiety rather showed lower levels of capacity impairment. 
Table 2 Correlations of different work-anxieties with capacity impairments $(N=244)$. Spearman correlations.

\begin{tabular}{|c|c|c|c|c|c|c|c|}
\hline Capacity impairment & $\begin{array}{c}\text { Adjustmen } \\
\mathrm{t} \text { disorder } \\
\text { with } \\
\text { anxiety }\end{array}$ & $\begin{array}{c}\text { Specific } \\
\text { Social } \\
\text { Anxietie } \\
\text { s }\end{array}$ & $\begin{array}{l}\text { Situatio- } \\
\text { nal } \\
\text { Anxiety }\end{array}$ & $\begin{array}{c}\text { Hypocho } \\
\text { n-driac } \\
\text { Anxiety }\end{array}$ & $\begin{array}{c}\text { Anxiety of } \\
\text { Insuffi- } \\
\text { ciency }\end{array}$ & $\begin{array}{c}\text { Generalized } \\
\text { Worrying }\end{array}$ & $\begin{array}{l}\text { Work- } \\
\text { place } \\
\text { Phobia }\end{array}$ \\
\hline Adherence to regulations & .045 & $.147 *$ & -.067 & $.135 *$ & .119 & -.088 & $.220 * *$ \\
\hline Planning and structuring tasks & .027 & .055 & -.085 & .108 & $.197 * *$ & $.197 * *$ & $.184 * *$ \\
\hline Flexibility & .120 & .056 & -.089 & $.145^{*}$ & .079 & .113 & $.244 * *$ \\
\hline Applying expertise & .113 & .074 & $-.141 *$ & .060 & $.156^{*}$ & -.007 & $.285^{* *}$ \\
\hline Capacity to judge and decide & .041 & .039 & $-.149 *$ & .121 & .113 & $.242 * *$ & $.271 * *$ \\
\hline Endurance & .064 & -.010 & .111 & .032 & $.240 * *$ & -.066 & .088 \\
\hline Assertiveness & .108 & $.490 * *$ & $-.178 * *$ & -.003 & .126 & -.111 & $.267 * *$ \\
\hline Contacts with others & .032 & $.264 * *$ & $-.254 * *$ & .022 & $.216^{* *}$ & $-.193 * *$ & .086 \\
\hline Teamwork capacity & .047 & $.361 * *$ & $-.229 * *$ & .035 & $.136^{*}$ & $-.155^{*}$ & $.167 *$ \\
\hline Self-care & -.004 & -.007 & $-.228 * *$ & $.154^{*}$ & $.261 * *$ & .037 & $.160^{*}$ \\
\hline Mobility & .061 & $.161 *$ & -.018 & -.004 & $.144^{*}$ & .071 & $.289 * *$ \\
\hline Familiar and intimate relationships & .067 & .040 & -.126 & .063 & .078 & .129 & $.139 *$ \\
\hline Non-work activities & .099 & .075 & -.078 & .118 & $.205 * *$ & $.176^{* *}$ & $.198 * *$ \\
\hline
\end{tabular}



Correlations of capacity impairment with later sick leave duration in patients with different work-anxieties $(N=244)$.

\begin{tabular}{|c|c|c|c|c|c|c|c|c|}
\hline Capacity impairment & $\begin{array}{c}\text { Adjustmen } \\
\text { t disorder } \\
\text { with } \\
\text { anxiety } \\
(n=32) \\
\end{array}$ & $\begin{array}{c}\text { Specific } \\
\text { Social } \\
\text { Anxietie } \\
\text { s } \\
(n=63)\end{array}$ & $\begin{array}{c}\text { Situatio- } \\
\text { nal } \\
\text { Anxiety } \\
(n=92)\end{array}$ & $\begin{array}{c}\text { Hypocho } \\
\text { n-driac } \\
\text { Anxiety } \\
(n=18)\end{array}$ & $\begin{array}{l}\text { Anxiety of } \\
\text { Insuffi- } \\
\text { ciency } \\
(n=70)\end{array}$ & $\begin{array}{c}\text { Generalized } \\
\text { Worrying } \\
\quad(n=90)\end{array}$ & $\begin{array}{l}\text { Work- } \\
\text { place } \\
\text { Phobia } \\
(n=29)\end{array}$ & $\begin{array}{c}\text { All } \\
\text { patients } \\
\text { with work- } \\
\text { anxieties } \\
(N=244)\end{array}$ \\
\hline Adherence to regulations & .354 & .173 & -.051 & .238 & .109 & .074 & .294 & .109 \\
\hline Planning and structuring tasks & .337 & .227 & -.046 & .025 & .061 & .094 & .141 & .085 \\
\hline Flexibility & .287 & .061 & -.009 & -.166 & .023 & .140 & .213 & .081 \\
\hline Applying expertise & .291 & $.254^{*}$ & $.219 *$ & .247 & .233 & .210 & .158 & $.268 * *$ \\
\hline Capacity to judge and decide & $.494 * *$ & .201 & .146 & .174 & .166 & $.294 * *$ & .32 & $.202 * *$ \\
\hline Endurance & -.015 & .010 & .138 & -.463 & -.037 & -.027 & -.223 & .056 \\
\hline Assertiveness & .269 & $.320 *$ & .120 & .046 & $.258^{*}$ & .085 & .225 & $.165^{*}$ \\
\hline Contacts with others & .105 & .246 & .192 & .202 & .219 & $.218^{*}$ & .263 & $.173 * *$ \\
\hline Teamwork capacity & .227 & .183 & .191 & .215 & .232 & .161 & .196 & $.160^{*}$ \\
\hline Self-care & .328 & $.392 * *$ & $.293 * *$ & .284 & $.319 *$ & $.278^{* *}$ & .295 & $.285^{* *}$ \\
\hline Mobility & .198 & .094 & .059 & .194 & -.081 & .084 & .206 & .105 \\
\hline Familiar and intimate relationships & -.082 & $.281 *$ & .075 & -.061 & .003 & .092 & -.139 & .114 \\
\hline Non-work activities & .272 & .073 & $.231 *$ & .058 & -.008 & .137 & .011 & $.149^{*}$ \\
\hline $\begin{array}{l}\text { Specific workplace-related work ability } \\
\text { prognosis by physician in hours }\end{array}$ & -.277 & -.217 & -.169 & -.105 & -.138 & -.206 & -.170 & $-.172 * *$ \\
\hline $\begin{array}{l}\text { General work ability prognosis by physician in } \\
\text { hours }\end{array}$ & -.055 & -.030 & -.093 & .260 & -.108 & -.133 & -.037 & -.104 \\
\hline Presently obtaining workplace ${ }^{1}$ & -.229 & -.118 & -.026 & .276 & .162 & .041 & -.245 & -.046 \\
\hline Mental work ability impairment ${ }^{2}$ & -.001 & .156 & .090 & .042 & .137 & .085 & .018 & .102 \\
\hline Physical work ability impairment ${ }^{2}$ & -.133 & .097 & .147 & .031 & .068 & -.009 & -.072 & $.131 *$ \\
\hline $\begin{array}{l}\text { Sick leave duration in the past } 12 \text { months in } \\
\text { weeks }\end{array}$ & .043 & .126 & .183 & 031 & .143 & .077 & .178 & $.146^{*}$ \\
\hline Gender $^{3}$ & -.004 & -.073 & .029 & -.007 & -.097 & -.092 & .146 & -.019 \\
\hline Age & -.088 & -.067 & .124 & .196 & .019 & -.051 & -.057 & .053 \\
\hline
\end{tabular}


Notes: ${ }^{1}$ Presently obtaining a workplace: $0=$ no workplace, $1=$ presently obtaining a workplace. ${ }^{2}$ Mental and physical work ability impairment: $0=$ no impairment, $1=$ work-relevant chronic impairment. ${ }^{3}$ Gender: $0=$ man, $1=$ woman. ${ }^{*} p<.05, * * p<.01$. Reading example: In patients with work-related adjustment anxiety, higher impairment in the capacity to judge and decide goes along with longer sick leave duration $\left(r=.494^{* *}\right)$. 
Table 3 shows the correlations between work capacity impairment and sick leave duration in the following six months after assessment. The physicians' global work ability prognosis and work ability impairment statements were hardly correlated with later sick leave duration. In contrast, some of the capacity dimensions were even specifically related to following sick leave. E.g. in patients with social anxiety higher impairment of assertiveness was systematically associated with longer duration of sick leave; in patients with generalized worrying, the degree of impairment in decision making and judgment was related with the duration of sick leave. Interestingly, impairment in general self-care was also significantly correlated with sick leave duration, and this was true in several work-anxieties (social anxiety, situational anxiety, insufficiency, worrying).

\section{Discussion}

First, results of this study show that patients with different work-anxieties differ in degree and pattern of work capacity impairment. The type of capacity impairment corresponds partly to the phenomenology of the anxiety, e.g. social anxiety goes along with impairment in interactional capacities. The specificity of capacity impairment in some of the anxieties is due to the fact that anxiety is partly a stimulus-bound phenomenon: social anxiety for example becomes specifically relevant in social interaction demands at work, but has not systematically to do with work contents, or tasks, or expertise and knowledge. Generalized worrying, which is phenomenologically a formal thought disorder with exaggerated negative thought fluency (Shearer and Gordon 2006), is accompanied with higher impairment in capacity to judge and decide. This is 
understandable, as decision making and judgment demands at work interfere with a psychopathology of formal thought disorder. Anxiety of insufficiency, which means a feeling of being not good enough and not fulfilling the work demands or work pace, mainly affects the endurance in a clinically relevant degree. However, it should be noticed that especially endurance shows a clinically relevant impairment degree in several work-anxieties.

We also found that some work-anxieties were not specifically related with circumscribed work capacity impairments. Situational anxiety for example can be associated with various circumscribed stimuli, and different people may perceive anxiety in different work situations or towards different work capacity demands. Situational anxiety (in opposite to a rather circumscribed social anxiety) can be learned in the sense of conditioned anxiety after any threatening event at work. Situational anxiety may also arise when the person perceives competency deficits, e.g. anxiety towards a new computer-program s/he cannot cope with (Top and Yilmaz 2015). Therefore it is likely that there are no salient impairments of specific capacity dimensions in patients with situational anxiety. This is also the case for workplace phobia. Workplace phobia is a phobic reaction towards the complex workplace as a whole. Thereby different capacity dimensions may be impaired. The only common characteristic of workplace phobia is that affected persons get physiological arousal when coming near their workplace and therefore they avoid the workplace. This results in an impairment to approach the workplace, i.e. capacity impairment in mobility. But beside, patients with workplace phobia also had higher capacity impairment in several other dimensions. This means that workplace phobia is rather quantitatively different from the other work-anxieties, in the sense that it goes along with even stronger 
impairment in different capacity dimensions.

The second important finding is that in patients with different workanxieties, different capacity impairments are associated with later sick leave duration: In patients with social anxiety, the impairment of assertiveness is related with sick leave duration, in patients with generalized worrying, the impairment of decision and judgment capacity is associated with sick leave duration. These correlations are stronger as when using global measures of work ability (here: physicians overall socio-medical judgments). This shows that a differential diagnostic of work capacities (instead of a global rating on work ability impairment) is useful in order to describe more concretely what the person cannot do and why s/he may be on sick leave.

An astonishing result was that (contrarily to other studies, Baron and Linden, 2009) that self-care impairment was correlated with sick leave duration quite consistently. An explanation may be that self-care is not only needed for general life, but also in most occupations, e.g. when contacts with thirds or teamwork is needed. Self-care thus might appear as a basic capacity for work ability which is required in most jobs. Therefore also higher self-care impairment may be associated with longer sick leave duration. A methodological explanation is that capacity impairment in this present study was rated specifically with reference to the work context, and not concerning the more global context of general life (as has been done by Baron and Linden 2009).

\section{Limitations}


A limitation for the interpretation of the results is the phenomenon of workanxiety comorbidity. In about half of the sample different work-anxieties occur as comorbid phenomena. This means that work-anxieties are usually not to be found as purely alone-standing symptoms, but may also occur as a complex syndrome. Therefore impairment degrees shown for the single work-anxiety diagnosis may be partly confounded with capacity impairment due to another comorbid workanxiety symptom.

No causal interpretations can be derived from these data: It cannot be said whether a primary state of work-anxiety causes the work capacity impairment or whether a reduced capacity level (due to missing training or competency or other illness) leads to work-anxiety.

Implications

In clinical practice, both symptomatology (work-anxiety) and capacity impairment must be seen as interacting phenomena, but finally capacity disorders rather than symptoms explain participation disorders and sick leave (Baron and Linden 2009; Gatchel et al. 1994). Interventions however can always target the capacity level of the person (beside treatment of symptomatology) (Muschalla 2014). Interventions can also be conducted at the workplace, either in terms of capacity trainings (e.g. stress management, Limm et al. 2011), or in terms of work design and workplace adjustment according to the capacity impairment level of employees (van Ruitenbeek et al. 2013).

According to our findings, training of endurance seems of importance in several work-anxieties. Stepwise occupational reintegration, i.e. re-entering the workplace with reduced working hours for a certain time, may be a useful 
temporary workplace adjustment means (Bürger \& Streibelt, 2011).

In persons with work-related social anxiety, training of interactional capacities may be done, or social exposition at work may be reduced (workplace adjustment). Persons with exaggerated work-related worrying may be trained in decision making and judgment, or their work tasks may be adjusted so that they are confronted with easier or fewer decision demands.

Further research may use the capacity concept as a basis for more differentiatedly describing work impairment, or as an outcome for training or workplace adjustment interventions.

\section{Key points}

Different dimensions of work capacity impairment (e.g. endurance, social interaction capacities, decision making and judgment, mobility) are of importance in different workanxieties. The capacity concept is useful to describe work-impairment clinically more differentiated than a global score of work ability impairment. On this basis more effective means for reintegration - capacity training or work adjustment - can be found. 


\section{Acknowledgements}

This research has been financially supported by the German Pension Fund. Grant number: $8011-106-31 / 31.107$

\section{Conflicts of interest}

There are no conflicts of interest.

\section{Informed consent and ethical standard}

Patients participated in this study with written informed consent.

All procedures performed in this study involving human participants were in accordance with the ethical standards of the institutional and/or national research committee and with the 1964 Helsinki declaration and its later amendments or comparable ethical standards. The study was approved by the ethics committee of the University of Potsdam. 


\section{References}

Balestrieri M, Isola M, Bonn R, Tam T, Vio M, Linden M, Maso E (2013) Validation of an Italian version of the Mini-ICF-APP, a short instrument for rating activity and participation restrictions in psychiatric disorders. Epidemiol Psychiatric Sci 22:81-91

Baron S, Linden M (2009) Disorders of functions and disorders or capacity in relation to sick leave in mental disorders. Int J Soc Psychiatr 55:57-63

Boschman JS, van der Molen HF, Frings-Dresen MH, Sluiter JK (2014) The impact of common mental disorders on work ability in mental and physically demanding construction work. Int Arch Occup Environ Health 87:51-59

Bürger W, Streibelt M (2011) Who benefits from stepwise occupational reintegration provided under the statutory pension insurance scheme? Rehabilitation 50:178-185

Dell-Kuster S, Lauper S, Koehler J, Zwimpfer J, Altermatt B, Zwimpfer T, Zwimpfer L, Young J, Bucher HC, Nordmann AJ (2014) Assessing work ability - a cross sectional study of interrater agreement between disability claimants, treating physicians, and medical expert. Scand J Work Environ Health 40:493-501

DGPM Deutsche Gesellschaft für Psychosomatische Medizin und Ärztliche Psychotherapie e.V., DKPM Deutsches Kollegium für Psychosomatische Medizin (2012) Leitlinie zur Begutachtung psychischer und psychosomatischer Erkrankungen. Sk2-Leitlinie, AWMF- 
Leitlinien-Register Nr. 051/029, Stand: 31.03 .2012 (gültig bis: 31.03.2017). Online available: www.awmf.org

DRV Deutsche Rentenversicherung Bund (2012) Leitlinien für die sozialmedizinische Begutachtung. Sozialmedizinische Beurteilung bei psychischen und Verhaltensstörungen. DRV, Berlin

Ekberg K, Wahlin C, Persson J, Bernfort L, Öberg B (2015) Early and Late return to Work after Sick Leave: Predictors in a Cohort of Sick-Listed Individuals with Common Mental Disorders. J Occup Rehabil, in press.

Esposito E, Wang JL, Williams JV, Patten SB (2007) Mood and anxiety disorders, the association with presenteeism in employed members of a general population. Epidemiol Psichiatr Soc 16:231-237

Fleishman EA, Reilley ME (1992) Handbook of Human Abilities: Definitions, measurements, and job task requirements. Consulting Psychologists Press, Palo Alto, CA

French, J.R.P. Jr. (1973). Person role fit. Occupational Mental Health 3:15-20

Gatchel RJ, Polatin PB, Mayer TG, Garcy PD (1994) Psychopathology and the rehabilitation of patients with chronic low back pain disability. Arch Phys Med Rehabil 75:666-670

Haines J, Williams CL, Carson JM (2002) Workplace Phobia: Psychological and psychophysiological Mechanisms. Int J Stress Manage 9:129-145 
Hannula JA, Lahtela K, Järvikoski A, Salminen JK, Mäkela P (2006) Occupational Functioning Scale (OFS) - An instrument for assessment of work ability in psychiatric disorders, Nord J Psychiatry 60:372-378

Hensing G, Bertilsson M, Ahlborg G, Waern M, Vaez M (2013) Self-assessed mental health problems and work capacity as determinants of return to work: a prospective general population-based study of individuals with all-cause sickness absence. BMC Psychiatry $13: 259$

Huijs JJ, Koppes LL, Taris TW, Blonk RW (2012) Differences in predictors of return to work among long-term sick-listed employees with different self-reported reasons for sick leave. J Occup Rehabil 22:301-311

Lidwall U (2015) Sick leave diagnoses and return to work: a Swedish register study. Disabil Rehabil 37:396-410

Limm H, Gündel H, Heinmüller M, Marten-Mittag B, Nater UM, Siegrist J, Angerer P (2011) Stress management interventions in the workplace improve stress reactivity: a randomized controlled trial. Occup Environ Med 68:126-133

Linden M, Baron S, Muschalla B (2010) Capacity according to ICF in relation to work related attitudes and performance in psychosomatic patients. Psychopathology 43:262-267 
Linden M, Baron S, Muschalla B, Ostholt-Corsten M (2015) Fähigkeitsbeeinträchtigungen bei psychischen Erkrankungen. Diagnostik, Therapie und sozialmedizinische Beurteilung in Anlehnung an das Mini-ICF-APP. Hogrefe, Göttingen

Linden M, Muschalla B (2007) Anxiety disorders and workplace-related anxieties. J Anxiety Disord 21:467-474

Molodynski A, Linden M, Juckel G, Yeeles K, Anderson C, Vazquez-Montes M, Burns T (2013) The reliability, validity, and applicability of an English language version of the MiniICF-APP. Soc Psychiatry Psychiatr Epidemiol 48:1347-1354

Muschalla B (2014) Fähigkeitsorientierte Verhaltenstherapie bei psychischen Erkrankungen. Verhaltenstherapie 24:48-55

Muschalla B, Fay D, Jöbges M, Linden M, Ayhan H, Flöge B, Heidrich ML (2014)

Evaluation einer Gruppentherapie für arbeitsplatzbezogene Ängste und Arbeitsplatzphobie. Abschlussbericht zum DRV-Forschungsprojekt. Brandenburgklinik Bernau und Universität Potsdam, Arbeits- und Organisationspsychologie

Muschalla B, Heldmann M, Fay D (2013) The significance of job-anxiety in a working population. Occup Med 63:415-421

Muschalla B, Linden M (2009) Workplace Phobia - A first explorative study on its relation to established anxiety disorders, sick leave, and work-directed treatment. Psychol Health Med $14: 591-605$ 
Muschalla B, Linden M (2013). Arbeitsplatzbezogene Ängste und Arbeitsplatzphobie. Phänomenologie, Differentialdiagnostik, Therapie, Sozialmedizin. Kohlhammer-Verlag, Stuttgart

Peterson NG, Borman WC, Hanson MA, Kubisiak UC (1999) Summary of results, implications for O*NET applications and future directions. In Peterson NG, Mumford MD, Borman WC, Jeanneret PR, Fleishman EA (Eds) An occupational information system for the $21^{\text {st }}$ century. American Psychological Association, Washington, DC, pp. 289-296

Schneider W, Henningsen P, Dohrenbusch R, Freyberger HJ, Irle H, Köllner V, Widder B (Eds) (2012) Begutachtung bei psychischen und psychosomatischen Erkrankungen. Autorisierte Leitlinien und Kommentare. Verlag Hans Huber, Bern

SGVP Schweizer Gesellschaft für Versicherungspsychiatrie (2012) Qualitätsleitlinien für psychiatrische Gutachten in der Eidgenössischen Invalidenversicherung. Schweizerische Gesellschaft für Psychiatrie und Psychotherapie SGPP, Bern

Shearer S, Gordon L (2006) The Patient with Excessive Worry. American Family Physician 73:1049-1056

Sheehan D, Janavs J, Baker R, Knapp E, Sheehan KH, Sheehan M (1994) MINI. Mini International Neuropsychiatric Interview. University of South Florida, Tampa Stansfeld S, Feeney A, Head J, Canner R, North F, Marmot M (1995) Sickness absence for 
psychiatric illness: the Whitehall II Study. Soc Sci Med 40:189-197

Stansfeld SA, Clark C, Caldwell T, Rodgers B, Power C (2008) Psychosocial work characteristics and anxiety and depressive disorders in midlife: the effects of prior psychological distress. Occup Environ Med 65: 634-642

Szubert Z, Sobala W (2002) Health reasons for firefighters to leave their job. Med Pr 53:291298.

Tuomi K, Ilmarinen J, Jahkola A, Katajarinne L, Tulkki A (1998) Work Ability Index, 2nd revised edition. Finnish Institute of Occupational Health, Helsinki

Top M, Yilmaz A (2015) Computer anxiety in nursing: an investigation from Turkish nurses. J Med Syst 39:163

Van Ruitenbeek G, Mulder MJGP, Zijlstra FRH, Nijhuis FJ, Mulders HPG (2013) An alternative approach for work redesign: experiences with the method "Inclusive Redesign of Work Processes". Gedrag en Organisatie 23:104-122

Wahlin-Norgren C, Ekberg K, Öberg B (2011) Is an expert diagnosis enough for assessment of sick leave for employees with musculoskeletal and mental disorders? Disabil Rehabil $33: 1147-1156$

WHO World Health Organization (2001) International Classification of Functioning, Disability and Health (ICF). Geneva: World Health Organization. 
https://doi.org/10.24355/dbbs.084-201907231330-0 